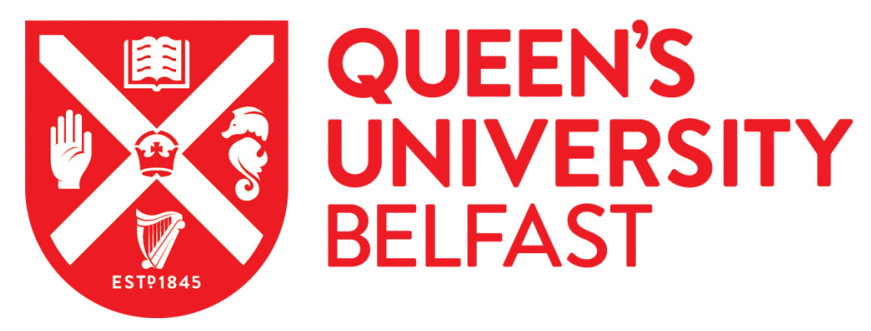

\title{
Identification and bioactivity evaluation of two novel temporins from the skin secretion of the European edible frog, Pelophylax kl.
} \section{Esculentus}

Chen, X., Wang, H., Yang, M., Wang, L., Zhou, M., Chen, T., \& Shaw, C. (2016). Identification and bioactivity evaluation of two novel temporins from the skin secretion of the European edible frog, Pelophylax kl. Esculentus. Biochemical and Biophysical Research Communications, 476(4), 566-573.

https://doi.org/10.1016/j.bbrc.2016.05.163

\section{Published in:}

Biochemical and Biophysical Research Communications

\section{Document Version:}

Peer reviewed version

Queen's University Belfast - Research Portal:

Link to publication record in Queen's University Belfast Research Portal

\footnotetext{
Publisher rights

(C) 2016 Elsevier B. V. This is an open access article published under a Creative Commons Attribution-NonCommercial-NoDerivs License (https://creativecommons.org/licenses/by-nc-nd/4.0/), which permits distribution and reproduction for non-commercial purposes, provided the author and source are cited.
}

\section{General rights}

Copyright for the publications made accessible via the Queen's University Belfast Research Portal is retained by the author(s) and / or other copyright owners and it is a condition of accessing these publications that users recognise and abide by the legal requirements associated with these rights.

Take down policy

The Research Portal is Queen's institutional repository that provides access to Queen's research output. Every effort has been made to ensure that content in the Research Portal does not infringe any person's rights, or applicable UK laws. If you discover content in the Research Portal that you believe breaches copyright or violates any law, please contact openaccess@qub.ac.uk. 


\section{Identification and bioactivity evaluationof two novel temporins from the}

skin secretionof the European edible frog, Pelophylax kl.esculentus

Xiaole Chen ${ }^{1, *}$, He Wang ${ }^{2, *}$, Mu Yang ${ }^{3}$, Lei Wang ${ }^{3}$, Mei Zhou ${ }^{3}$, Tianbao Chen ${ }^{3}$, Chris Shaw ${ }^{3}$

1. School of Pharmacy, Fujian Medical University, Fuzhou, Fujian, China

2. School of Integrative Medicine, Fujian University of Traditional Chinese Medicine, China

3. MedicineNatural peptide discovery group, School of Pharmacy, Queen's University, Belfast BT9 7BL, Northern Ireland, UK

* Corresponding author. Tel: +86(0)15806076835; Fax:+86(0)591 22862016

E-mail address: leochen5139@fjmu.edu.cn (Xiaole Chen); hwang11@qub.ac.uk (He Wang) 


\begin{abstract}
Two novel members of the temporin family, named temporin-1Ee

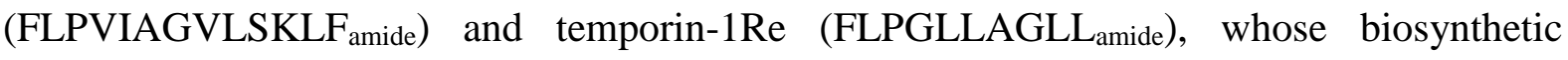
precursor structures were deduced from clones obtained from skin secretion-derived cDNA libraries of the European edible frog, Pelophylax kl. esculentus, were obtained by 'shotgun' cloning. Deduction of the molecular masses of each mature processed peptide from respective cloned cDNAs was used to locate respective molecules in reverse-phase HPLC fractions of secretion. Temporin-1Ee $(\mathrm{MIC}=10 \mu \mathrm{M})$ and temporin-1Re $(\mathrm{MIC}=60 \mu \mathrm{M})$ were both found to be active against Gram-positive Staphylococcus aureus, but retaining a weak haemolytic activity.Single-site substitutions can dramatically change the spectrum of activity of a given temporin. Compared with temporine-1Ec, just one chemically-conservative substitution (Val8 instead of Leu8), temporin-1Ee bearing a net charge of +2 displaysbroadspectrum activity with particularly high potency on the clinically relevant Gram-negative strains, Escherichia coli $(\mathrm{MIC}=40 \mu \mathrm{M})$. These factors bode well for translatingtemporinsto bepotential anti-infective drug candidates.
\end{abstract}


Keywords:Amphibian; Antimicrobial Peptide; Temporins;Cloning

\section{Introduction}

Temporins, containing 10-13 amino acid residues, are considered to be among the smallest AMPs found in Nature. They were initially described as the Vespa-like peptides, due to their sequence similarities with chemotactic and histamine-releasing peptides isolated from the venom of wasps of the genus Vespa, and were first identified in the skins of the European edible frog, Pelophylax kl. esculentus and the Asian frog, Rana erythraea. Afterwards, a family of 10 , structurally-related peptides with antibacterial and antifungal properties were isolated from the electrically-stimulated skin secretions of the European common red frog, Rana temporaria, and called temporins A to L, in reflection of their source (1). Compared with other ranid frog skin AMPs that have a C-terminal heptapeptide ring constructed by a disulphide bridge, the temporins are $\mathrm{C}$-terminally $\alpha$-amidated and stabilised by helix-forming residues such as leucine, alanine and lysine. Due to their basic residues, temporins usually carry a net charge ranging from 0 to +4 at neutral $\mathrm{pH}$ and have a potent activity against Gram-positive bacteria including methicillin-resistant S. aureus (MRSA) with little toxicity to mammalian cells $(2,3)$. However, since temporin $\mathrm{L}$ is the firstmember of this family isolated which exhibit broad spectra of activity against both Gram-positive and Gramnegative bacteria (Giacometti et al 2006), a few new members of the temporin family, such as temporin-Sha, temporin-T1 and temporin-1DRa, have been found to display similar antimicrobial activitiesto temporine $\mathrm{L}(4,5)$.

Naturally occurring AMPs differ dramatically in various interrelated peptide parameters, including size, net charge, hydrophobicity, amphipathicity, degree of structure formation and flexibility (6-8), thus the selectivity and mechanism of antimicrobial activitiesremain unknown.It is well accepted that differences in lipid composition of membranes among microorganisms play key roles in the selectivity of AMPs toward microbial cells (2), and 
although the mechanism of action of temporinshave been only scarcely evaluated, most are supposed to have a classical membrane perturbing activity through a barrel-stave mechanism of action. In contrast, temporin $\mathrm{L}$ is presumed to insert into bacterial cell membranes through the formation of pore-like openings and interact with essential anionic targets inside the cells (1-3, 9-10). Temporins as short chain peptides of simple structure are considered to be good templates for new drug design.

Herein, two novel antimicrobial peptides have been identified in the skin secretion of the European Edible frog, Pelophylax kl. esculentus, through initial molecular cloning of skin secretion-derived cDNAs. Deduced peptides were subsequently identified in fractions of skin secretion, chemically-synthesised and subjected to functional analyses. Sequence characterisation of the mature peptides demonstrated that they belonged to the temporin family found predominantly in the skin secretions of ranid frogs. Thus, these two peptides were named temporin-1Ee and temporin-1Re. Temporin-1Ee, which is an analogue of temporin-1Ec, has broad-spectrum antimicrobial activity at low micromolar concentrations. Temporin-1Re, with a net charge of +1 , is weakly active against the Gram-positive bacterium, $S$. aureus, but is devoid of activity against Gram-negative bacteria and yeast. 


\section{Materials and methods}

\section{Preparation of $\boldsymbol{P}$. kl.esculentusskin secretion}

P.kl.esculentus $(n=30)$ were obtained from a commercial source (Le QuerruyCellier, Notre Dame de Monts, France) and had been captive bred. The frogs were adults on receipt and were settled into their new surroundings for 3 months prior to secretion harvesting. They were maintained in our purpose-designed amphibian facility at $15-20^{\circ} \mathrm{C}$ under a $12 \mathrm{~h} / 12 \mathrm{~h}$ light/dark cycle and fed multivitamin-loaded crickets three times per week. Skin secretion was obtained from dorsal skin by mild transdermal electrical stimulation $(5 \mathrm{~V} ; 3 \mathrm{~ms}$ pulses $)$ for $30 \mathrm{~s}$. The skin secretion was washed from the skin using deionized water, snap-frozen in liquid nitrogen, and lyophilized. Lyophilizate was stored at $-20{ }^{\circ} \mathrm{C}$ prior to analysis.

\section{“Shotgun" cloning of Pelophylax kl. esculentus skin secretion-derived cDNA}

Five milligrams of lyophilised skin secretion were dissolved in $1 \mathrm{ml}$ lysis/binding buffer (Dynal, UK) to stabilise mRNA. Polyadenylated mRNA was trapped and isolated by means of magnetic oligo-dTDynabeads as described by the manufacturer (Dynal, UK). The trapped template polyadenylated mRNA was employed to manufacture a cDNA library using reverse transcriptase and a sample of this library was subjected to 5'- and 3'-rapid amplification of cDNA ends (RACE) procedures to obtain full-length antimicrobial peptide precursor nucleic acid sequence data using a SMART-RACE kit (Clontech UK) following manufacturer's instructions. Briefly, the 5'-RACE reactions employed the forward primer (5'ATGTTCACCATGAAGAAATC-3') and the reverse primer (5'-CTATCCCACATC AGGAGACTTTCC-3') were designed according to the nucleotide sequence of temporin precursor cDNAs obtained from other ranid frogs. The 5'-RACE reactions were purified and cloned using a pGEM-T vector system (Promega Corporation) and sequenced using an ABI 3730 automated sequencer (Applied Biosystems, Foster City, CA, USA). 


\section{Reverse phase HPLC fractionation of skin secretion}

A further 1mg sample of lyophilised skin secretion was dissolved in $0.5 \mathrm{ml}$ of $0.05 / 99.5$ (v/v) trifluoroacetic acid (TFA)/water and clarified of microparticulates by centrifugation. The supernatant was then subjected to HPLC using a gradient formed from 0.05/99.5 (v/v) TFA/water to $0.05 / 29.95 / 70.0(\mathrm{v} / \mathrm{v} / \mathrm{v}) \mathrm{TFA} / \mathrm{water} /$ acetonitrile in $240 \mathrm{~min}$ at a flow rate of 1 ml/min. A Cecil Adept Binary HPLC system (Adept Technology, Inc. USA), fitted with an analytical column (a Jupiter 00G4052 semi-preparative C-5 column 25x1cm, Phenomenex, U.K.) was employed and this was interfaced with a Thermo Fisher Scientific LCQ Fleet electrospray ion-trap mass spectrometer. The effluent from the chromatographic column was flow-split with approximately $10 \%$ entering the mass spectrometer source and $90 \%$ directed towards a fraction collector. Dead volume between column and fraction collector was minimal $(20 \mu 1)$. The molecular masses of polypeptides in each chromatographic fraction were further analysed using matrix-assisted laser desorption/ionisation, time-of-flight mass spectrometry (MALDI-TOF MS) on a linear time-of-flight Voyager DE mass spectrometer (Perseptive Biosystems, MA, USA) in positive detection mode using alpha-cyano-4hydroxycinnamic acid as the matrix. Internal mass calibration of the instrument with known standards established the accuracy of mass determination as $\pm 0.1 \%$.

\section{Peptide synthesis and purification}

Following unequivocal establishment of the primary structure of natural temporin-1Ee and temporin-1Re, replicates were synthesised by solid-phase Fmoc chemistry using a PS3 automated peptide synthesiser (Protein Technologies, Tucson, AZ, USA). When the synthesis cycles were completed, the peptides were cleaved from the resins using 95/2.5/2.5 (v/v/v) TFA/TIPS/water for $6 \mathrm{~h}$, precipitated in ether over the next $24 \mathrm{~h}$, washed exhaustively in six changes of ether and then allowed to completely dry over a further $24 \mathrm{~h}$. The peptides were then dissolved in a minimal quantity of $0.05 / 99.5, \mathrm{v} / \mathrm{v}, \mathrm{TFA} / \mathrm{water}$, snap frozen in liquid 
nitrogen and lyophilised. Degree of purity and authentication of structures of the synthetic peptides were determined using MALDI-TOF MS as previously described.

\section{Antimicrobial assays}

Antimicrobial activities of synthetic temporin-1Ee and temporin-1Re were assessed by determination of minimal inhibitory concentrations (MICs) using a standard Gram-positive bacterium S. aureus - NCTC 10788), a standard Gram-negative bacterium (Escherichia coli-NCTC 10418) and a standard pathogenic yeast (Candida albicans NCPF 1467). Temporin-1Ee and temporin-1Re were tested within the concentration range of $160-2.5 \mu \mathrm{M}$ and were initially dissolved as a stock solution of $200 \mu \mathrm{M}$ in sodium phosphate-buffered saline, $\mathrm{pH}$ 7.2, and subsequently diluted in Mueller-Hinton broth (MHB). Peptide concentrations in the range stated, were inoculated with microorganism cultures (105 colony forming units $(\mathrm{CFU}) / \mathrm{ml})$, and placed into 96-well microtiter cell culture plates. Plates were incubated for $18 \mathrm{~h}$ at $37^{\circ} \mathrm{C}$ in a humidified atmosphere. Following this, the growth of bacteria/yeast was determined by means of measuring optical density (OD) at $\lambda=550 \mathrm{~nm}$ by an ELISA plate reader (BioliseBioTek EL808). Minimal inhibitory concentrations (MICs) were defined as the lowest concentration at which no growth was detectable. 


\section{Results}

\section{Molecular cloning of the A biosynthetic precursor}

Two different cDNAs, each encoding a novel peptide, named temporin-1Ee and temporin-1Re, respectively, were consistently and repeatedly cloned from the library during RACE-PCR experiments (Figure1). Such experiments used $R$. esculenta skin mRNA as a template and specific oligonucleotides, designed to a conserved region (Signal peptide and 3'-UTR) of previously cloned preprotemporincDNAs from other ranid species. The cDNAs amplified were separated on agarose gels then isolated with the cloning vector which was transformed into host cells. The white and drug resistant bacterial colonies were chosen by random and subjected to nucleotide sequence analysis. Alignment of temporin-1Ee and -1Re nucleotide sequences and open-reading frame amino acid sequences, using the AlignX programme of the Vector NTI Bioinformatics suite (Informax), revealed a very high degree of primary structural similarity of both nucleic acid and amino acid sequence between the temporin-1Ee and -1Re (Figure 2).An NCBI BLAST search found that the novel temporin1Ee showed at least $92 \%$ sequence identity with temporin-1Ec and peptide A1 and peptide B9 obtained from $R$. esculenta, previously (Table 2). In the precursor sequence, the first 22 amino acid residues encoded a putative signal peptide at the $\mathrm{N}$-terminal followed by an acidic amino acid residue rich spacer peptide consisting of 21 amino acids for temporin-1Ee and 22 amino acids for temproin-1Re with a pair of classical basic residue -Lys-Arg- (-K-R-) propeptide convertase cleavage sites at its C-terminus. A single copy of a mature temporin sequence was found at the C-terminus just upstream of a Gly residue that serves as an amide donor for the C-terminal residue of the temporin. The nucleotide sequence of the temporin1Ee and temporine-1Re precursors from the skin secretion of the European edible frog, Pelophylax kl.esculentus, have been deposited in the EMBL Nucleotide Sequence Database under the accession code KT437658 and KT437659. 
Identification and structural analysis of mature temporin-1Ee and temporin-1Re in skin secretion fractions and subsequent chemical synthesis

The two novel temporins (temporin-1Ee: FLPVIAGVLSKLF-amide and temporin-1Re: FLPGLLAGLL-amide ), deduced from cloned skin cDNAs, with computed molecular mass of $1402.08 \mathrm{Da}$ and $1012.32 \mathrm{Da}$, respectively, were identified in HPLC fraction numbers 164 and 143, respectively (Figures 3 and 4). Both temporins were successfully obtained by the solid-phase chemical synthesis. The reverse phase HPLC chromatogramsobtained from each synthesis following cleavage from the resin and deprotection, showed major peaks in each case representing approximately $95 \%$ of the synthetic mixture and these were subjected to MALDI-TOF mass analysis. In each case, molecular ions consistent with predicted molecular masses of respective novel temporinswere observed indicating successful syntheses and confirming a high degree of purity $(>95 \%)$ of the expected products.

\section{Antimicrobial/hemolytic activity oftemporin-1Ee and temporin-1Re}

The abilities of temporin-1Ee and temporin-1Re peptides to inhibit the growth of the tested microorganisms employed are compared in Table 3. MICs obtained against S. aureus were $10 \mu \mathrm{M}$ for temporin-1Ee and $60 \mu \mathrm{M}$ for temporin-1Re. Moreover, temporin-1Ee displayed an inhibitory effect on the growth of the Gram-negative bacterium,E.coli, MIC was $40 \mu \mathrm{M}$, whereas temporin-1Re exhibit no effect. Both temporins were ineffective in inhibiting the growth of the yeast, $C$. albicans, at concentrations up to and including $160 \mu \mathrm{M}$. Moreover,temporin-1Ee was found to possess no haemolytic activity at the MIC $(10 \mu \mathrm{M})$ determined for S. aureus. This experiment was repeated five times. 


\section{Discussion}

The AMPs stored in the glands of frog skin display a complicated array of typical peptide physico-chemical attributes related to size, charge, hydrophobicity, degree of secondary structure formation and amphipathicity. Temporins have been intensely-studied due to their potent activity against Gram-positive bacteria, including clinical isolates of methicillin-resistant Staphylococcus aureus and vancomycin-resistantEntercoccusfaeciumand Enterococcus faecalis $(9,12)$. However, due to their small size and low charge, the antimicrobial activity mechanisms of temporins are still unclear. The most recent research has provided some new insights to explain the mechanism of action of the temporins. Take temporin- $\mathrm{L}$ as an example. Temporin- $\mathrm{L}$ is a 13-mer peptide in which a tail-to-tail dimerisation can occur that then suits the barrel-stave model, binding to the vesicles surface, and undergoing a rapid local aggregation that causes a reorientation. Membrane insertion steps can only occur after this oligomerisation (12-14). We presume that temporin-1Ee, which has the same chain length as temporin-L and also possesses antimicrobial activity against both Gram-negative and Gram-positive bacteria, may act via a similar mechanism in which the carpet model could explain this temporin's antimicrobial activity but the barrelstave model could explain the haemolytic effect $(1-3,15)$.

Hence the variation in net charges $(0$ to +3$)$, chain length (generally $10-14$ residues), and amino acid sequences could influence the efficacy and selectivity of temporins against pathogens. Most members of the temporin family carry a low net charge of 0 or +1 at physiological $\mathrm{pH}$ and their chain lengths are restricted and small. Within the temporins, the higher the net positive charge $(+2$ or +3$)$, the more potent they are in killing bacteria. Temporin-1Ee, with a net charge of +2 , is more potent $(\mathrm{MIC} 10 \mu \mathrm{M})$ than temporin-1Re (MIC $60 \mu \mathrm{M})$, with a net charge of +1 , in killing the model Gram-positive bacterium, Staphylococcus aureus (NCTC 10788). In contrast, due to most of the natural 
temporinshaving a weak charge and small size, they generally have little or no effects on Gram-negative bacteria, fungi and yeasts (16-20). However, one of the prototype temporins, temporin L (13 residues, net charge +3 ), was found to exhibit broad-spectrum activity against many microorganisms, including Gram-positive bacteria, Gram-negative bacteria and yeast, even cancer cells and human erythrocytes. Many additional temporins subsequently discovered exhibit broad-spectrum antimicrobial activity, examples being temporin-1Sa (11) and temporin-1DRa (21-22). Temporin-1Ee, with a net charge of +2 , has efficacy and a high degree of activity against the model Gram-positive bacterium, S. aureus, with an MIC close to that of temporin-1Ec (MIC $8 \mu \mathrm{M})$ (23). However temporin-1Ee, which has an amino acid sequence quite similar to that of temporin-1Ec with just one chemically-conservative substitution (Val8 instead of Leu8 - Table 2.), is able to inhibit the growth of the model Gram-negative bacterium, Escherichia coli. Thus even conservative, single-site substitutions can dramatically change the spectrum of activity of a given temporin. The temporins and their analogues are thus well accepted candidates for the design of new antimicrobial peptides for reasons such as above, where small changes in amino acid sequence can result in dramatic improvements in antimicrobial activity but retaining a weak haemolytic activity (24-27). These factors bode well for translating temporins into clinically useful anti-infective agents to combat antibiotic-resistant pathogens. 


\section{Acknowledgments}

This work was supported by National Natural Science Foundation, China (Grant No. 81402842), Natural Science Foundation of Fujian Province, China (Grant No. 2015J05162), Natural Science Foundation of Fujian Province, China (Grant No. 2016J01374), Fujian Provincial outstanding talents for Education and Scientific research (Grant No. JK2014016) and TheDoctor StartupFoundation of Fujian Medical University (Grant No.2012bs002).

\section{Conflict of Interest statement}

The authors declare that they have no conflict of interest.

\section{Ethical statement}

All applicable international, national, and/or institutional guidelines for the care and use of animals were followed. 


\section{Data deposition footnotes}

The nucleotide sequence of the temporin-1Ee and temporine-1Re precursors from the skin secretion of the European edible frog,Pelophylax kl.esculentus, have been deposited in the EMBL Nucleotide Sequence Database under the accession code KT437658 and KT437659. 


\section{References}

1.Simmaco, M., Mignogna, G., Canofeni, S., Miele, R., Mangoni, M. L., \& Barra, D. (1996). Temporins, antimicrobial peptides from the European red frog, Rana temporaria.European Journal of Biochemistry / Febs, 242, 3, 788-92.

2. Mahalka, A. K., \&Kinnunen, P. K. J. (2009). Binding of amphipathic helical antimicrobial peptides to lipid membranes: Lessons from temporins B and L. BiochimicaEtBiophysicaActa - Biomembranes, $1788,8,1600-1609$.

3. Simmaco, M., Mignogna, G., Barra, D., \&Bossa, F. (1994). Antimicrobial peptides from skin secretions of Pelophylax kl.esculentus. Molecular cloning of cDNAs encoding esculentin and brevinins and isolation of new active peptides.The Journal of Biological Chemistry, 269, 16, 1195661.

4. F. Abbassi, B. Oury, T. Blasco, D. Sereno, G. Bolbach, P. Nicolas, K. Hani,M. Amiche, A. Ladram, Isolation, characterization and molecular cloning ofnew temporins from the skin of the North African ranidPelophylaxsaharica,Peptides 29 (2008) 1526e1533.

5. E. Urban, E. Nagy, T. Pal, A. Sonnevend, J.M. Conlon, Activities of four frog skinderivedantimicrobial peptides (temporin-1DRa, temporin-1Va and themelittin-related peptides AR-23 and RV-23) against anaerobic bacteria, Int. J.Antimicrob. Agents 29 (2007) $317 \mathrm{e} 321$

6. J.M. Conlon, N. Al-Ghaferi, B. Abraham, J. Leprince, Strategies for transformationof naturally-occurring amphibian antimicrobial peptides intotherapeutically valuable antiinfective agents, Methods 42 (2007) 349e357.

7. C. El Amri, P. Nicolas, Plasticins: membrane-damaging peptides with 'chameleon-like' properties, Cell.Mol. Life Sci. 65 (2008) 895e909.

8. P. Joanne, M. Falord, O. Chesneau, C. Lacombe, S. Castano, B. Desbat,C. Auvynet, P. Nicolas, T. Msadek, C. El Amri, Comparative study of twoplasticins: specificity, interfacial behavior, and bactericidal activity,Biochemistry 48 (2009) 9372e9383. 
9.Giacometti, A., Cirioni, O., Ghiselli, R., Mocchegiani, F., Orlando, F., Silvestri, C., Bozzi, A., Rinaldi, A. C. (2006). Interaction of Antimicrobial Peptide Temporin L with Lipopolysaccharide In Vitro and in Experimental Rat Models of Septic Shock Caused by Gram-Negative Bacteria. Antimicrobial Agents and Chemotherapy, 50, 7, 2478.

10. Giacometti, A., Cirioni, O., Kamysz, W., D'Amato, G., Silvestri, C., Del, P. M. S., Licci, A., Scalise, G. (2005). In vitro activity and killing effect of temporinA on nosocomial isolates of Enterococcus faecalis and interactions with clinically used antibiotics. Journal of Antimicrobial Chemotherapy, 55, 2, 272-274.

11. Abbassi, F., Oury, B., Blasco, T., Sereno, D., Bolbach, G., Nicolas, P., Hani, K., Ladram, A.(2008). Isolation, characterization and molecular cloning of new temporins from the skin of the North African ranid, Pelophylaxsaharica. Peptides, 29, 9, 1526-33.

12. Carotenuto, A., Malfi, S., Saviello, M. R., Campiglia, P., Gomez-Monterrey, I., Mangoni, M. L., Gaddi, L. M. H., Grieco, P. (2008). A Different Molecular Mechanism Underlying Antimicrobial and Hemolytic Actions of Temporins A and L. Journal of Medicinal Chemistry, 51, 8, 2354.

13.Zhao, H., \&Kinnunen, P. K. (2002). Binding of the antimicrobial peptide temporin L to liposomes assessed by Trp fluorescence.The Journal of Biological Chemistry, 277, 28, 25170-7.

14. Zhao, H., Rinaldi, A. C., Di, G. A., Simmaco, M., \&Kinnunen, P. K. (2002). Interactions of the antimicrobial peptides temporins with model biomembranes.Comparison of temporins B and L. Biochemistry, 41, 13, 4425-36.

15. Papo, N., \& Shai, Y. (2003). Exploring peptide membrane interaction using surface plasmon resonance: differentiation between pore formation versus membrane disruption by lytic peptides. Biochemistry, 42, 2, 458-66.

16. Mangoni, M. (2006). Temporins, anti-infective peptides with expanding properties. Cellular and Molecular Life Sciences, 63, 9, 1060-1069.

17. Mangoni, M. L., \& Shai, Y. (2009). Temporins and their synergism against Gram-negative bacteria and in lipopolysaccharide detoxification.BiochimicaEtBiophysicaActa, 1788, 8, 1610-9. 
18. Simonetti, O., Cirioni, O., Goteri, G., Ghiselli, R., Kamysz, W., Kamysz, E., Silvestri, C., Scalise, A. (2008). Temporin A is effective in MRSA-infected wounds through bactericidal activity and acceleration of wound repair in a murine model. Peptides , 29, 4, 520-528.

19. Wade, D., Silberring, J., Soliymani, R., Heikkinen, S., Kilpelainen, I., Lankinen, H., \&Kuusela, P. (2000). Antibacterial activities of temporinAanalogs. FEBS Letters, 479, 1, 6.

20. Wade, D., Silveira, A., Silberring, J., Kuusela, P., \&Lankinen, H. (2000). Temporin Antibiotic Peptides: A Review and Derivation of a Consensus Sequence. Protein and Peptide Letters, 7, 349-358.

21. Conlon, J. M., Al-Ghafari, N., Coquet, L., Leprince, J., Jouenne, T., Vaudry, H., \& Davidson, C. (2006). Evidence from peptidomic analysis of skin secretions that the red-legged frogs, Rana aurora draytonii and Rana aurora aurora, are distinct species. Peptides, 27, 6, 1305-1312.

22. Conlon, J. M., Kolodziejek, J., \&Nowotny, N. (2004). Antimicrobial peptides from ranid frogs: taxonomic and phylogenetic markers and a potential source of new therapeutic agents. BiochimicaEtBiophysicaActa, 1696, 1, 1-14.

23. Ali, M. F., Knoop, F. C., Vaudry, H., \& Conlon, J. M. (2003). Characterization of novel antimicrobial peptides from the skins of frogs of the Pelophylax kl.esculentus complex. Peptides, 24, 7, 955-961.

24. ukala, T. L., Bowie, J. H., Maselli, V. M., Musgrave, I. F., \& Tyler, M. J. (2006). Host-defence peptides from the glandular secretions of amphibians: structure and activity. Natural Product Reports, $23,3,368-393$.

25. Saviello, M. R., Malfi, S., Campiglia, P., Cavalli, A., Grieco, P., Novellino, E., \&Carotenuto, A. (2010). New insight into the mechanism of action of the temporin antimicrobial peptides. Biochemistry, 49, 7, 1477-85.

26. I. Zelezetsky, A. Tossi, Alpha-helical antimicrobial peptides: using a sequencetemplate toguide structure-activity relationship studies, Biochim. Biophys.Acta 1758 (2006) $1436 \mathrm{e} 1449$. 
27. Koczulla, A. R., \& Bals, R. (2003). Antimicrobial Peptides: Current Status and Therapeutic Potential. Drugs, 63, 4, 389-406.

\section{Legends to Figures}

Figure 1.Nucleotide sequences of cloned cDNAs encoding the biosynthetic precursors of temporin1Ee (A) and -1Re (B) and translated amino acid sequences of their open-reading frames. Putative signal peptides are double-underlined, mature peptides are single-underlined and the stop codons are indicated by asterisks. The $\mathrm{G}$ residue (italics) at the $\mathrm{C}$-terminal of the mature peptide sequence serves as an amide donor after removal of the Lys residue (italics) with a carboxypeptidase. Residues G or GK (italics) at the end of the mature peptide sequence serve for C-terminal amidation.

Figure 2.(A) Alignment of full-length nucleotide sequences of clones encoding the precursors of temporin-1Ee and temporin-1Re. DNA sequences were copied in FASTA format, which were then subjected to multiple sequence alignment by the CLUSTALW program. (B) Alignment of openreading frame amino acid sequences of preprotemporin-1Ee and preprotemporin-1Re. The conserved residues are shaded. Amino acid sequences were copied in FASTA format, which were then subjected to multiple sequence alignment by the CLUSTALW program.

Figure 3.Reverse phase HPLC chromatogram of the skin secretion from Rana esculenta with arrows indicating elution positions/retention times of the two novel antimicrobial peptides, temporin-1Re and temporin-1Ee. The $\mathrm{Y}$-axis indicates absorbance units at $\lambda=214 \mathrm{~nm}$.

Figure 4. (A) MALDI-TOF mass spectrum showing major peptide molecular ion in reverse phase HPLC fraction $\sim 164$ of Pelophylax kl. esculentus skin secretion corresponding in mass to temporin-1Ee (1402.08Da). (B) MALDI-TOF mass spectrum of peptide molecular ion in reverse phase HPLC fraction $\sim 143$ of skin extract corresponding to temporin-1Re (1012.32Da). 


\section{Figure 1}

(A)

\begin{tabular}{lllllllllllllllll}
$M$ & $\mathrm{~F}$ & $\mathrm{~T}$ & $\mathrm{M}$ & $\mathrm{K}$ & $\mathrm{K}$ & $\mathrm{S}$ & $\mathrm{L}$ & $\mathrm{L}$ & $\mathrm{L}$ & $\mathrm{L}$ & $\mathrm{F}$ & $\mathrm{F}$ & $\mathrm{L}$ & $\mathrm{G}$ & $\mathrm{T}$ & $\mathrm{I}$ \\
\hline
\end{tabular}

1 ATGTTCACCA TGAAGAAATC CCTGTTACTC CTTTTCTTCC TTGGGACCAT TACAAGTGGT ACTTCTTTAG GGACAATGAG GAAAAGAAGG AACCCTGGTA

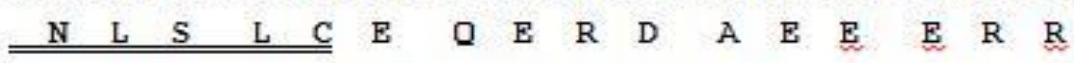

51 CAACTTATCT CTCTGTGAGC AAGAGAGAGA TGCTGAGGAA GAAAGAAGAG GTTGAATAGA GAGACACTCG TTCTCTCTCT ACGACTCCTT CTTTCTTCTC \begin{tabular}{lllllllllllllllll}
$D$ & $D$ & $P$ & $E$ & $E$ & $R$ & $D$ & $V$ & $E$ & $M$ & $E$ & $K$ & $R$ & $F$ & $L$ & $P$ & $V$ \\
\hline
\end{tabular} 101 ACGATCCAGA AGAAAGGGAT GTTGAAATGG AAAAACGATT TTTACCAGTC TGCTAGGTCT TCTTTCCCTA CAACTTTACC TTTTTGCTAA AAATGGTCAG

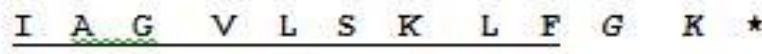

151 ATTGCTGGAG TTTTGAGTAA ACTGTTCGGC AAATAACCCA AAAATGTTGA TAACGACCTC AAAACTCATT TGACAAGCCG TTTATTGGGT TTTTACAACT 201 AACTTTGGAA ATAGAATTGG AAATCATCTG ATGTGGAATA TCATTTAGCT TTGAAACCTT TATCTTAACC TTTAGTAGAC TACACCTTAT AGTAAATCGA

251 AAATGCACAA CAGATGTCTT ATAAAAAAAT AAAGATATCA CATATAAAAA TTTACGTGTT GTCTACAGAA TATTTTTTTA TTTCTATAGT GTATATTTTT

301 AAAAAAAAAA AAAAAAAAAA AAAAA TTTTTTTTTT TTTTTTTTTT TTTTT

(B)

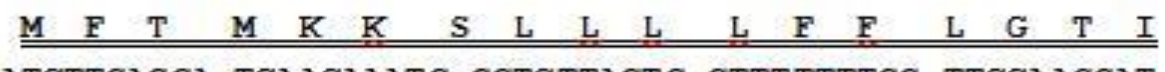

1 ATGTTCACCA TGAAGAAATC CCTGTTACTC CTTTTTTTCC TTGGAACCAT TACAAGTGGT ACTTCTTTAG GGACAATGAG GAAAAAAAGG AACCTTGGTA

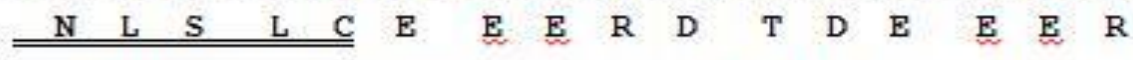

51 CAACTTATCC CTTTGTGAGG AAGAGAGAGA TACTGATGAG GAAGAAAGAA. GTTGAATAGG GAAACACTCC TTCTCTCTCT ATGACTACTC СTTCTTTCTT \begin{tabular}{llllllllllllllllll}
$R$ & $D$ & $E$ & $P$ & $D$ & $E$ & $M$ & $D$ & $V$ & $E$ & $V$ & $E$ & $K$ & $R$ & $F$ & $L$ & $P$ \\
\hline
\end{tabular}

101 GAGATGAGCC AGATGAAATG GATGTTGAAG TGGAAAAACG ATTTTTACCA. CTCTACTCGG TCTACTTTAC CTACAACTTC ACCTTTTTGC TAAAAATGGT

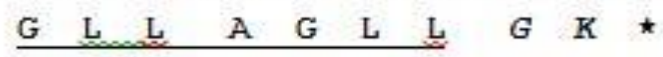

151 GGTCTGCTTG CTGGTTTGTT GGGAAAGTAA CCAAAAATGT TGAAACTTTG CCAGACGAAC GACCAAACAA CCCTTTCATT GGTTTTTACA ACTTTGAAAC 201 GAAATGGAAA AGGAAATCAT CTGATGTGGA ATATCATTTA GCTAAATGCT CTTTACCTTT TCCTTTAGTA GACTACACCT TATAGTAAAT CGATTTACGA 251 TAACAGATGT CTTTATAAAA AATAAATAAA TATGTCGCAT AAGAAAGGCC ATTGTCTACA GAAATATTTT TTATTTATTT ATACAGCGTA TTCTTTCCGG

301 CAAAAAATAA AAAACCCAAA AaAAAAATTT TTAAAAgAaA AAA GTTTTTTATT TTTTGGGTTT TTTTTTTAAA AATTTTCTTT TTT 


\section{Figure 2}

(A)

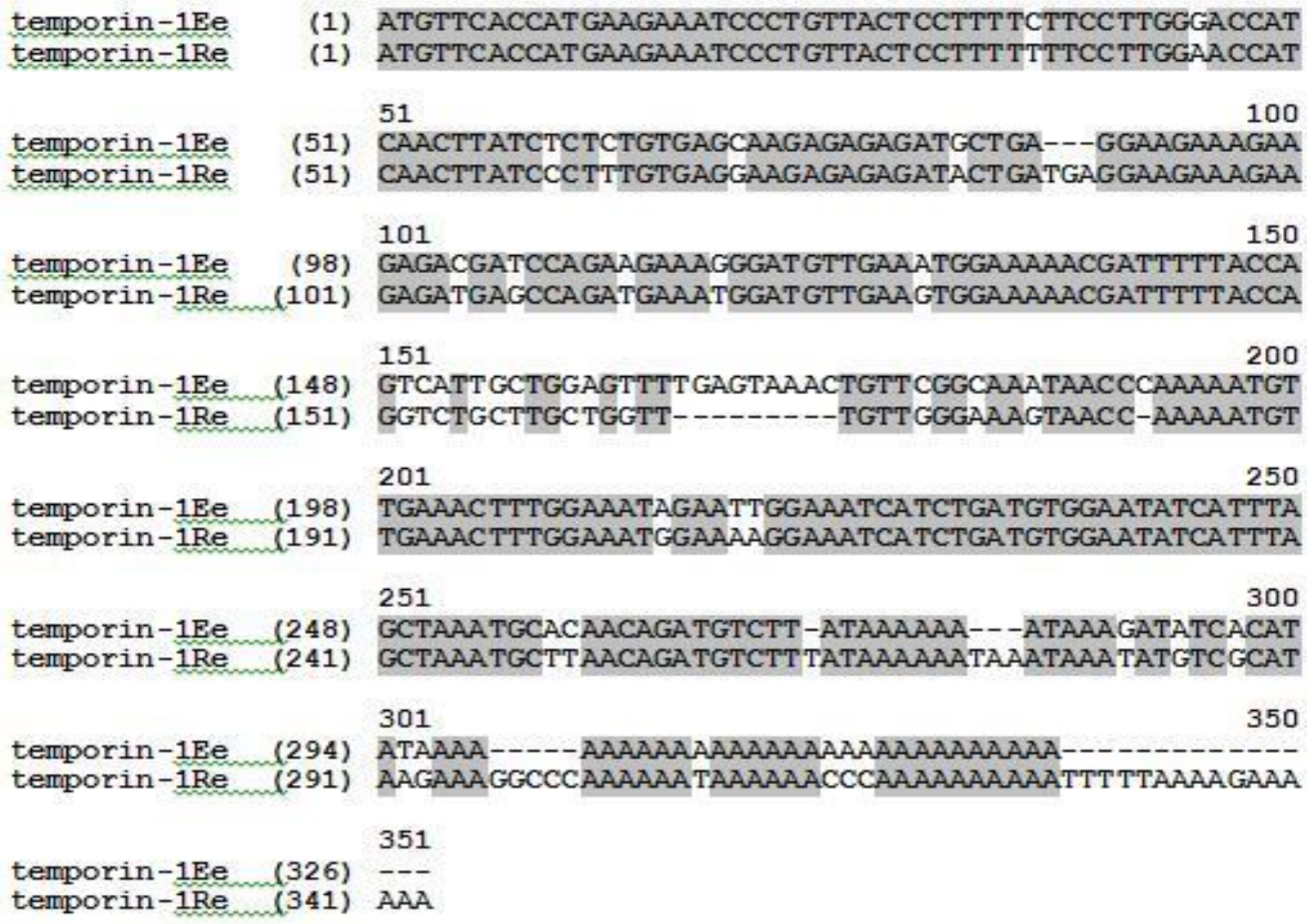

(B)

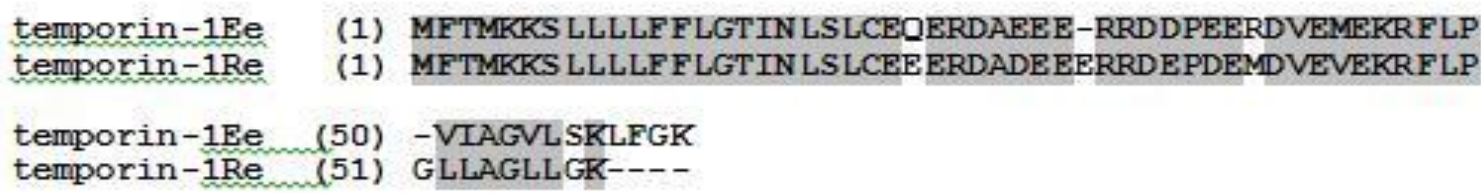


Figure 3

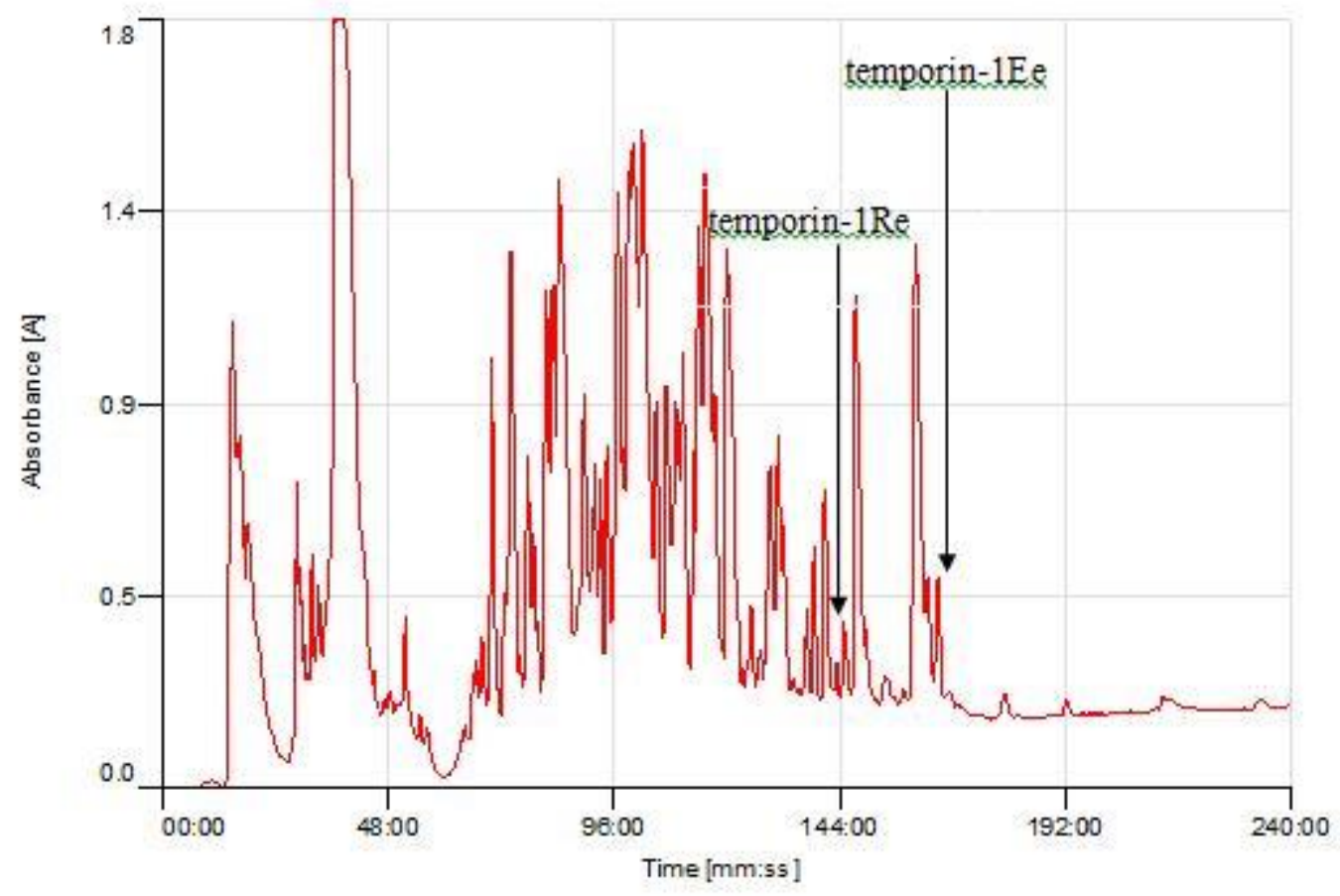


Figure 4

(A)

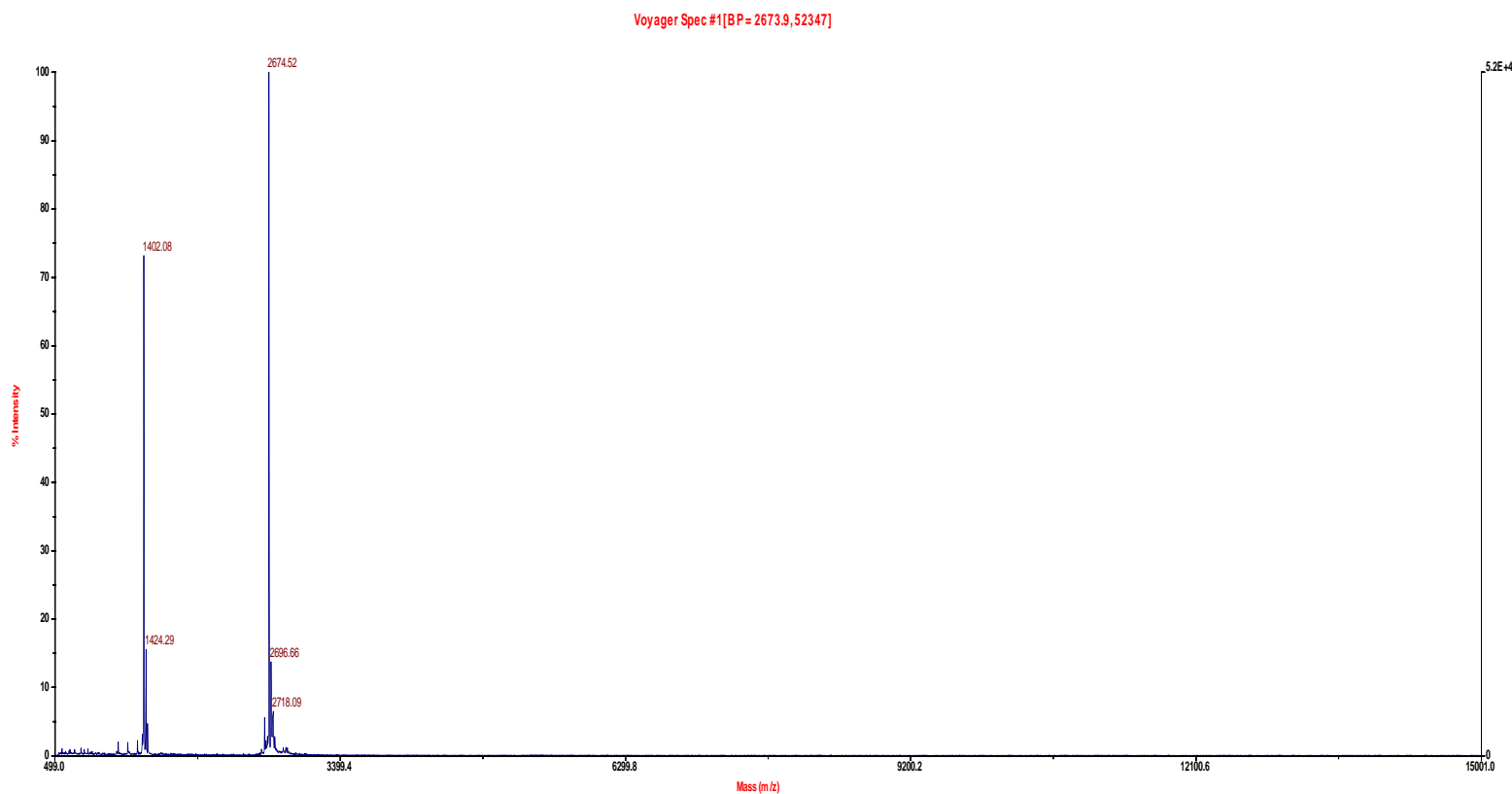

(B)

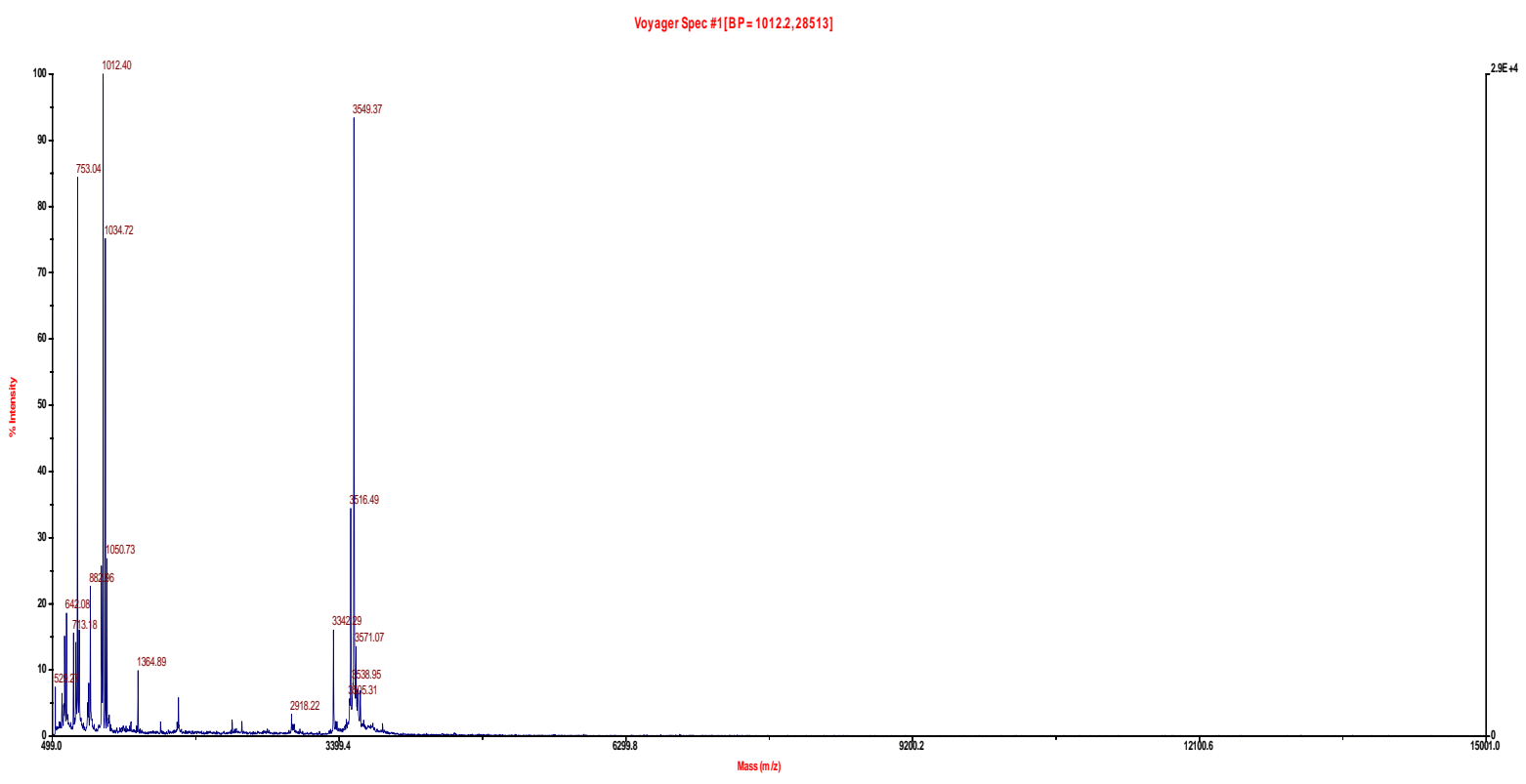

